\title{
ANNOUNCEMENT OF THE PROJECT FOR THE COMPUTATION OF MATHEMATICAL TABLES
}

\author{
FEDERAL WORKS AGENCY \\ WORK PROJECTS ADMINISTRATION \\ NEW YORK, N. Y.
}

Sponsored by the Bureau of Standards, Washington, D. C., Dr. Lyman J. Briggs, Director, and Dr. Arnold N. Lowan, Technical Director of the Project. Administrative Staff: Mr. Murray Pfeferman and Mr. Milton Abramowitz. Technical Staff: Dr. Gertrude Blanch, Dr. Norman Davids, Mr. William Horenstein, Mr. Jacob L. Miller, Miss Ida Rhodes, Dr. J. Rosenthal, and Mr. Herbert E. Salzer.

The Project for the Computation of Mathematical Tables has been in operation since January 1, 1938. The aim of this project is to compute Mathematical Tables of fundamental importance in Mathematics, Physics, Chemistry, Engineering, Statistics and related sciences. Lists of (A) Tables Published, (B) Tables in Process of Reproduction, (C) Tables for Which Manuscripts are Completed, (D) Tables for Which Computations are Completed, (E) Tables for Which Computations are in Progress and $(\mathrm{F})$ Tables under Consideration, are given in this announcement.

The project would welcome suggestions for the computation of tables of interest in pure and applied Mathematics as well as information regarding computational work in progress elsewhere. Communications should be addressed to Oliver A. Gottschalk, Acting Administrator, Work Projects Administration, 70 Columbus Avenue, New York City.

Requests for copies of published tables should be addressed to Dr. Lyman J. Briggs, Director, National Bureau of Standards, Washington, D. C.

\section{A. Tables Published}

1. Table of the First Ten Powers of the Integers from 1 to 1,000. 80 pp. (1939).

2. Tables of the Exponential Function $e^{x} .535$ pp. (1939).

$$
\begin{array}{rlrl}
x & =[-2.5000(0.0001) 1.0000 ; 18 D], 1 & x & =[1.0000(0.0001) 2.5000 ; 15 D], \\
x & =[2.500(0.001) 5.000 ; 15 D], & x & =[5.00(0.01) 10.00 ; 12 D] .
\end{array}
$$

3. Tables of $\sin x$ and $\cos x$ for Radian Arguments. 275 pp. (1940).

$$
\begin{array}{lll}
x=[0.000(0.001) 25.000 ; 8 D], & x=[0(1) 100 ; 8 D], \\
x=[0.00001(0.00001) 0.00009 ; 15 D], & x=[0.0001(0.0001) 0.0009 ; 15 D], \\
x=[0.001(0.001) 0.009 ; 15 D], & x=[0.01(0.01) 0.09 ; 15 D], \\
x=[0.1(0.1) 0.9 ; 15 D], & x=[0.00000(0.00001) 0.01000 ; 12 D] .
\end{array}
$$

There is also included a conversion table between radians and degrees.

4. Tables of $\sin x, \cos x, \sinh x, \cosh x$ for Radian Arguments. 405 pp. (1940).

$$
x=[0.0000(0.0001) 1.9999 ; 9 D], \quad x=[0.0(0.1) 10.0 ; 9 D] .
$$

There is also included a conversion table between radians and degrees.

5. Tables of Planck's Radiation and Photon Functions. Published in the Journal of the Optical Society of America, vol. 30, pp. 70-81 (1940).

1 The figures in the square brackets give the range and the interval of the argument, and the number of decimal places $(D)$ or significant figures $(S)$ in the entries. 
6. Table of $S i(x), C i(x), E i(x)$ and $-E i(-x)$, where

$$
\begin{aligned}
S i(x) & =\int_{0}^{x} \frac{\sin t}{t} d t, \quad C i(x)=\int_{\infty}^{x} \frac{\cos t}{t} d t, \\
E i(x) & =\int_{-\infty}^{x} \frac{e^{t}}{t} d t, \quad-E i(-x)=\int_{x}^{\infty} \frac{e^{-t}}{t} d t, \\
x & =[0.0000(0.0001) 2.0000 ; 9 D] .
\end{aligned}
$$

7. Table of $S i(x), C i(x), E i(x)$ and $-E i(-x)$,

$$
x=\left[0.000(0.001) 10.000 ; 9 S, 10 S_{\lrcorner} \cdot{ }^{1}\right.
$$

8. Tables of Grid Coordinates (American Polyconic Projection) at 5 minute intervals of latitude and longitude for latitudes from $7^{\circ}$ to $28^{\circ}$. (For the War Department.)

\section{B. Tables in Process of Reproduction}

1. Table of $\log _{e} N, N=[1(1) 100,000 ; 16 D]$.

2. Table of $\log _{e} x, x=[0.0000(0.0001) 10.0000 ; 16 D]$.

3. Table of the Probability Functions:

$$
\begin{array}{cl}
\frac{2}{\pi^{1 / 2}} e^{-x^{2}}, & \frac{2}{\pi^{1 / 2}} \int_{0}^{x} e^{-t^{2}} d t, \\
{[x=0.0000(0.0001) 1.0000 ; 15 D],} & x=[1.000(0.001) 5.736 ; 15 D] .
\end{array}
$$

4. Table of the Probability Functions:

$$
\begin{gathered}
\frac{1}{(2 \pi)^{1 / 2}} e^{-x^{2} / 2}, \quad \frac{1}{(2 \pi)^{1 / 2}} \int_{-x}^{x} e^{-t^{2} / 2} d t, \\
x=[0.0000(0.0001) 1.0000 ; 15 D], \quad x=[1.000(0.001) 7.800 ; 15 D] .
\end{gathered}
$$

5. Tables of Section Moduli and Moments of Inertia for Structural Members used in Naval Architecture. (For the Bureau of Marine Inspection and Navigation.)

6. Table of the Electronic Functions

$$
G=\frac{1}{\left(1-\beta^{2}\right)^{1 / 2}}, \quad V=\frac{m_{0} c^{2}}{e}(G-1) \quad \text { and } \quad H \rho=\frac{m_{0} c^{2}}{e} \beta G
$$

for $\beta$ ranging from 0 to 0.9999999990 at various intervals.

7. Table of $\operatorname{arc} \tan x$ for

$$
x=[0.000(0.001) 7.000(0.01) 50.00(0.1) 300.0(1) 2000(10) 10000 ; 12 D] .
$$

8. Table of Five-Point Lagrangian Interpolants for arguments ranging between 0 and 2, intervals of 0.001. (For the War Department.)

\section{Tables for which Manuscripts are Completed}

1. Table of the Bessel Functions $J_{0}(z)$ and $J_{1}(z)$ for complex arguments, $z=\rho e^{i \theta}$, for $\rho$ ranging from 0 to 10 at intervals of 0.01 and $\theta$ ranging from $0^{\circ}$ to $90^{\circ}$ at intervals of $5^{\circ}$, to 10 decimal places.

2. Table of the definite integrals:

$$
\begin{gathered}
A(k, n)=\int_{0}^{1} x^{k} \sin n \pi x d x \text { and } B(k, n)=\int_{0}^{1} x^{k} \cos n \pi x d x \text { for } k=0, \\
1,2, \cdots, 10 \text { and } n=1,2, \cdots, 100 \text { to } 15 \text { decimals. }
\end{gathered}
$$


3. Table of $\int_{0}^{x} J_{0}(t) d t$ from 0 to 10 at intervals of 0.01 to 10 places.

4. Table of exact values of five-point Lagrangian Interpolants. (For the War Department.)

\section{Tables for which Computations are Completed}

1. Table of the fractional powers $x^{y}$ for both $x$ and $y$ ranging from 0 to 1 at inter. vals of 0.01 and for other values of $x$ and $y$ to 15 decimal places.

2. Tables of Stellar Functions for "Point-Source" Models.

\section{E. Tables for which Computations are in Progress}

1. Bessel Functions of the fractional orders $\pm 1 / 3, \pm 2 / 3, \pm 1 / 4, \pm 3 / 4$ for real and pure imaginary arguments ranging from 0 to 10 at intervals of 0.01 to 10 decimal places.

2. Table of $Q_{n}(x)=(\pi / 2 x)^{1 / 2} J_{n+1 / 2}(x)$ for $n=1,2,3, \cdots, 20$ and $x$ ranging from 0 to 10 at intervals of 0.01 to 10 decimal places.

3. Table of the Bessel Functions $Y_{0}(z)$ and $Y_{1}(z)$ for the same complex arguments as in C.1.

4. Tables of $\tan x$ and $\cot x$ for $x$ ranging from 0 to 2 radians at intervals of 0.0001 .

5. Table of $Q_{n}(x)=(\pi / 2 x)^{1 / 2} J_{n+1 / 2}(x)$ for $n=-21,-20, \cdots, 0$ and $x$ ranging from 0 to 10 at intervals of 0.01 to 10 decimal places.

6. Tables of $\operatorname{Si}(x)$ and $C i(x)$ for $x$ ranging from 10 to 100 at intervals of 0.01 .

7. Various hydraulic tables based on Kutter's and Manning's formulae. (For the War Department.)

8. Table of the Associated Legendre Functions of the First and Second Kind, for $n$ ranging between 1 and 10 , and $m$ between 0 and 4; for arguments $x$ and $i x$ where $x$ ranges between 0 and 10 at intervals of 0.1 . Also corresponding values for half-integral values of $n$, and values of the functions for arguments in degrees. (Tabulation suggested by the National Defense Research Committee.)

9. Tables of Grid Coordinates of Northwestern Extension of U. S. (American Polyconic Projection. For the War Department.)

10. Table for Map Projections of Northwestern Extension of U. S. (American Polyconic Projection. For the War Department.)

11. Tables of Length of Meridional Arc at one-minute intervals. (For the War Department.)

\section{F. Tables under Consideration}

1. A 12 place table of Inverse Circular and Hyperbolic Functions other than arc $\tan x$.

2. Extensive tables of Elliptic Integrals and Elliptic Functions.

3. Table of the Gamma Function for complex arguments, $x+i y$, for both $x$ and $y$ ranging from 0 to 5 at intervals of 0.05 .

4. Table of the Error Functions for complex arguments.

5. The zeros of the Legendre Polynomials up to the 16 th order to 15 decimal places and the weight coefficients for Gauss' Mechanical Quadrature Formula.

6. Tables of the Confluent Hypergeometric Functions for selected values of the parameters.

7. Table of the integral $\int_{0}^{x} Y_{0}(t) d t$.

8. Tables of three-point, four-point, and six-point Lagrangian Interpolants.

9. Table of the first ten powers of the reciprocals of the integers from 1 to 1000 .

10. Table of solutions of Mathieu's Differential Equation. 